\title{
Arterial elasticity as a risk factor for early cardiovascular disease among testicular cancer survivors treated with platinum-based chemotherapy: a cross-sectional pilot study
}

This article was published in the following Dove Press journal: Vascular Health and Risk Management

\author{
Anne H Blaes' \\ Daniel A Mulrooney ${ }^{2}$ \\ Rachel Isaksson Vogel ${ }^{3}$ \\ Anna Solovey' \\ Robert Hebbel' \\ Bruce A Peterson' \\ Joseph P Neglia ${ }^{4}$ \\ Carter Biewen ${ }^{5}$ \\ Suma H Konety ${ }^{6}$ \\ Daniel A Duprez
}

'Division of Hematology/Oncology, University of Minnesota, Minneapolis, MN, USA; ${ }^{2}$ St Jude Children's Research Hospital, Memphis, TN, USA; ${ }^{3}$ Division of Gynecology/Oncology, University of Minnesota, MN, USA; ${ }^{4}$ Department of Pediatrics, University of Minnesota, MN, USA; ${ }^{5}$ Division of Pediatrics, University of California San Francisco, CA, USA; 'Division of Cardiology, University of Minnesota, MN, USA

Correspondence: Anne H Blaes Division of Hematology, Oncology and Transplantation, Department of Medicine, University of Minnesota, 420 Delaware Street, SE, MMC 480 Minneapolis, MN 55455, USA

$\mathrm{Tel}+\mathrm{I} 6126268138$

$\mathrm{Fax}+|612626| 44 \mid$

Email Blaes004@umn.edu
Purpose: Testicular cancer survivors who have received platinum-based chemotherapy are at risk for premature cardiovascular disease. The etiology of this risk is not well understood. This pilot study explores the impact of platinum-based chemotherapy on endothelial function. Methods: Testicular cancer survivors $<30$ years old at the time of diagnosis who received platinum-based chemotherapy between 2002 and 2012, as well as 17 similarly aged male controls, were identified. Consented subjects underwent vascular assessment using the HDI/PulseWave CR-2000 Cardiovascular Profiling System and the Endo-PAT2000 system. Biomarkers and functional test markers were compared among cases, controls, and a group of historical controls using two sided two-sampled $t$-tests and Wilcoxon rank-sum tests.

Results: Thirteen survivors with a median age of 30.2 years and body mass index of 27.3 were enrolled, along with 17 healthy controls with a median age of 27.1 years and body mass index of 24.8. Median time from chemotherapy was 4.7 (range: 0.8-14) years. There was no statistical difference in reactive hyperemia peripheral arterial tonometry ratio between cases and controls $(p=0.574)$. There was no statistical difference in small or large artery elasticity between cases and controls $(p=0.086)$ or between cases and historical controls $(p=0.729)$. There was also no statistical difference in the blood levels of circulating endothelial cells, von Willebrand factor, and vascular cell adhesion molecules. There was a trend toward increased metabolic syndrome in cases $(15 \%)$ as compared to recruited controls $(6 \%)$, though this difference was not statistically significant $(p=0.565)$.

Conclusion: Testicular cancer survivors have no clinically significant difference in endothelial function compared to controls 4 years after the completion of chemotherapy. Further research is needed to explore the secondary modifiable causes that may contribute to the risk of premature cardiovascular disease.

Keywords: vascular injury, testicular cancer, chemotherapy, cardio-oncology, cardiac injury, cardiac disease

\section{Background}

Testicular cancer is the most common malignancy among 15 - to 40 -year-old males. ${ }^{1}$ In the past 3 decades, the 5-year survival rates of testicular cancer have increased from $63 \%$ to $>90 \%{ }^{1,2}$ As cancer survivors are living longer, however, they are more at risk for subsequent therapy-related health complications. Recent studies have suggested that testicular cancer survivors are at risk for premature cardiovascular disease, myocardial infarction, and sudden death..$^{2-5}$ Among Dutch testicular cancer survivors, the prevalence 
of cardiovascular disease is estimated to be $18 \% 20$ years after completion of therapy. ${ }^{6,7}$ In evaluating 992 patients diagnosed in the UK between 1982 and 1992, at a median follow-up of 10.2 years, the relative risk of cardiac events was 2-3 folds higher for all survivors who received chemotherapy alone, radiation alone, or combined chemotherapy and radiation, as compared to those who received surgery alone. ${ }^{8}$ Other studies have suggested a 5.7-fold increased risk of cardiovascular disease in those receiving platinum-based chemotherapy as compared to those receiving surgery alone. ${ }^{5}$ The risk appears to be higher in nonseminomatous testicular cancer survivors.

The etiology of premature cardiovascular disease in testicular cancer survivors is not known.,.$^{3,10-12}$ It has been hypothesized that platinum-based chemotherapy may cause direct, diffuse endothelial damage. ${ }^{10}$ This damage is most notable in the first year after chemotherapy. ${ }^{13,14}$ Platinumbased chemotherapy has also been associated with components of metabolic syndrome: weight gain, hyperlipidemia, hypertension, and insulin resistance. ${ }^{5,6,9}$ Secondary endothelial damage from metabolic syndrome may also contribute to premature cardiovascular disease. ${ }^{11}$ In the present study, we sought to determine whether endothelial dysfunction underlies the etiology of premature cardiovascular disease among testicular cancer survivors. We hypothesized that testicular cancer survivors treated with chemotherapy could have a reduction in small artery elasticity (SAE), suggestive of early atherosclerotic disease, as compared to healthy, noncancerous controls. We, therefore, conducted a cross-sectional pilot study to assess endothelial function among testicular cancer survivors who received platinum-based chemotherapy as compared to healthy controls.

\section{Methods}

\section{Subjects}

Testicular cancer survivors $<30$ years of age at the time of diagnosis and who received platinum-based chemotherapy between 2002 and 2012 were identified at the Masonic Cancer Clinic, University of Minnesota in Minneapolis, MN, and Park Nicollet Health Services in Saint Louis Park, MN, USA. Eligible subjects had received platinum-based chemotherapy, were disease free, had been off therapy for at least 6 months, and were not currently on any ongoing myelosuppressive therapy or chemotherapy. Subjects with a cardiac history (myocardial infarction, congestive heart failure, or cardiac catheterization requiring intervention) were excluded. Subjects with a prior history of radiation therapy were excluded. Medical record abstraction for cases confirmed the diagnosis, stage at diagnosis, use of chemotherapy, use of radiation therapy, and personal medical history including history of cardiovascular diseases and medications.

\section{Ethical approval}

The University of Minnesota Institutional Review Board and Cancer Center Review Committee, as well as the Park Nicollet Institutional Review Board, approved this protocol. All patients provided written informed consent according to the Declaration of Helsinki.

All procedures performed in the studies involving human participants were in accordance with the ethical standards of the institutional and/or national research committee and with the 1964 Declaration of Helsinki and its later amendments or comparable ethical standards. This article does not contain any studies with animals performed by any of the authors.

\section{Controls}

Seventeen healthy controls from the Minneapolis and Saint Paul area without a history of myocardial infarction, congestive heart failure, or cardiac catheterization were enrolled. After reviewing our data and determining that $\sim 30 \%$ of our cases and $0 \%$ of our controls smoked and knowing that tobacco use can affect vascular disease, we chose to also compare SAE and large artery elasticity (LAE) data from the testicular cancer survivor cohort with a group of historical controls in which a similar number had smoked tobacco. The data from healthy, historical controls were obtained from the Hennepin County Medical Center and previously published. ${ }^{15}$ Subjects were matched by age, sex, and smoking status. Subjects with a history of diabetes, hepatitis C, myocardial infarction, congestive heart failure, or cardiac catheterization requiring intervention (stent placement and angioplasty) were excluded.

\section{Study recruitment}

Potential subjects were mailed a letter of recruitment inviting them to participate in the study and have been previously described. ${ }^{16}$ A follow-up phone call was placed to the subjects who did not respond. Consented subjects were invited to undergo vascular assessment and biomarker assessment. The consented subjects who completed the protocol received a $\$ 50$ gift card.

\section{Vascular assessment}

The vascular assessment testing procedure has been previously described. ${ }^{16}$ Testing was performed using the EndoPAT 2000 and HDI/PulseWave CR-2000 Cardiovascular Profiling System (Hypertension Diagnostic Inc, Eagan, MN, 
USA). LAE and SAE are derived from the diastolic pulse contour analysis based on a modified Windkessel model of circulation (model HDI/PulseWave CR-2000 Cardiovascular Profiling System). Before and during the waveform assessment, an automated oscillatory blood pressure (BP) measurement was taken on the contralateral arm. This system has been standardized and correlated with cardiovascular risk factors. Grey et $\mathrm{al}^{17}$ have demonstrated that SAE is predictive for coronary heart disease events and that LAE and SAE are predictive for heart failure events above and beyond arterial BP. ${ }^{18} \mathrm{~A}$ reduction in reactive hyperemia, assessed by the Endo-PAT ratio $(<1.67)$, has also been correlated with an increase in cardiac events independent of other traditional cardiac risk factors. ${ }^{19}$

The flow-mediated dilation examination required the patient to be supine and at rest. Subjects were fasting for 8 hours. All vasoactive medications were withheld for at least 4 half-lives, when possible. The SonoSite Ultrasound system was equipped with vascular software for 2-dimensional imaging, color and spectral Doppler, an internal electrocardiogram (ECG) monitor, and a high-frequency vascular transducer.

All study assessments were conducted in a specifically designed research space for clinical vascular assessments. Blood collection and vascular functional measurements were conducted by the same trained vascular technician. The pulse waveform assessments were performed in triplicate, and the mean (average of 3 values for each participant) LAE and SAE indices were used in statistical analyses.

\section{Biomarker assessment}

Biomarkers were assessed using blood obtained from a fasting draw. Assessments of biomarkers for lipids and inflammatory changes included serum glucose, total cholesterol, low-density lipoprotein cholesterol, high-density lipoprotein cholesterol, triglycerides (TGs), C-reactive protein, plasminogen activator 1, tissue-type plasminogen activator, circulating endothelial cells, vonWillebrand factor (vWF), soluble vascular cell adhesion molecule, soluble P-selectin, surface P-selectin, and surface vascular cell adhesion molecule. Blood samples were collected prior to vascular functional assessments.

\section{Statistical analysis}

Demographic and clinical characteristics of participants by group (cases, controls, and historical controls) were obtained. For triplicate measurements, the average value was used in analysis. Mean $\pm \mathrm{SD}$ or median and range of the biomarkers and the functional test measurements are presented as appropriate. Differences between young adult survivors of testicular cancer and both control populations (recruited controls and historical controls) were analyzed in terms of the biomarkers and function tests using $t$-tests assuming unequal variances and Wilcoxon rank-sum tests as appropriate. Data were analyzed using SAS version 9.3 (SAS Institute Inc, Cary, NC, USA). A $p$-value of 0.05 was considered statistically significant.

\section{Results}

\section{Demographic and clinical characteristics}

Seventy testicular cancer survivors diagnosed between 2002 and 2013 were identified. Of these, 14 consented to participate in the study, resulting in an overall participation rate of $20 \%$. One participant was subsequently removed from the analysis when it was determined that he had received radiation therapy alone and no chemotherapy, leaving a final cohort of 13 testicular cancer survivors. Median time between completion of platinum-based chemotherapy and study participation was 4.7 years. The majority of survivors had received combination chemotherapy with cisplatin, etoposide, and bleomycin, with only 1 survivor receiving single-agent carboplatin. Demographic and clinical characteristics of cases, recruited controls, and historical controls are presented in Table 1.

\section{Cases and recruited controls}

Mean age of cases was $30.2 \pm 4.6$ years as compared to $27.1 \pm 8.3$ years for controls $(p=0.2)$. Testicular cancer cases tended to be Caucasian $(92 \%$ vs $56 \%$; $p=0.044)$ and overweight in comparison to controls (mean body mass index [BMI] $27.3 \pm 8.0 \mathrm{~kg} / \mathrm{m}^{2}$ compared to $24.8 \pm 3.6 \mathrm{~kg} /$ $\left.\mathrm{m}^{2} ; p=0.31\right)$. There was no statistical difference in lipid profiles between cases and controls. In addition, mean systolic blood pressure was elevated in cases $(125 \pm 11 \mathrm{mmHg}$ vs $113 \pm 11 \mathrm{mmHg}, p=0.01)$. There was no difference in free testosterone ( $11.4 \mathrm{vs} 16.5 \mathrm{pg} / \mathrm{mL}, p=0.10)$ or total testosterone ( 4.0 vs $5.4 \mathrm{ng} / \mathrm{mL}, p=0.09)$ levels between cases and controls. Smoking status, however, between cases and controls differed markedly $(31 \%$ vs $0 \%, p=0.026)$. As a result of this difference in smoking status, we also compared cases with a historical control group that had a similar proportion of tobacco users. No other demographic or clinical differences between cases and historical controls achieved statistical significance. 
Table I Demographic and clinical characteristics of cases and controls at a median follow-up time of 4.7 years after completion of platinum-based chemotherapy

\begin{tabular}{|c|c|c|c|}
\hline Outcome* & Cases $(n=13)$ & Recruited controls $(n=17)$ & Historical controls $(n=16)$ \\
\hline Age, years & $30.2(4.6)$ & $27.1(8.6)$ & $34.5(6.8)$ \\
\hline \multicolumn{4}{|l|}{ Race/ethnicity, \% } \\
\hline White & 92.3 & $56.3^{\wedge}$ & $43.8^{\wedge}$ \\
\hline Others & 7.7 & 43.8 & 56.3 \\
\hline Smoker & 30.8 & $0^{\wedge}$ & 37.5 \\
\hline Body mass index, $\mathrm{kg} / \mathrm{m}^{2}$ & $27.3(8.0)$ & $24.8(3.6)$ & $27.8(4.8)$ \\
\hline Systolic blood pressure, $\mathrm{mmHg}$ & $124.5(10.9)$ & $1 \mathrm{I} 3.4(10.9)^{\wedge}$ & $127.5(12.7)$ \\
\hline Diastolic blood pressure, $\mathrm{mmHg}$ & $77.7(9.9)$ & $73.4(5.8)$ & $75.5(9.0)$ \\
\hline Testosterone, $\mathrm{ng} / \mathrm{mL}^{\wedge \wedge}$ & $4.0(2.5)$ & $5.4(1.6)$ & - \\
\hline Free testosterone, $\mathrm{pg} / \mathrm{mL}$ & II.4 (9.4) & $16.5(5.9)$ & - \\
\hline Total cholesterol, $\mathrm{mg} / \mathrm{dL}$ & $178.2(45.4)$ & $168.4(39.6)$ & $187.9(40.6)$ \\
\hline $\mathrm{HDL}, \mathrm{mg} / \mathrm{dL}$ & $43.0(15.6)$ & $47.4(9.4)$ & $51.4(13.6)$ \\
\hline $\mathrm{LDL}, \mathrm{mg} / \mathrm{dL}$ & $106.4(43.7)$ & $97.1(27.8)$ & $112.4(13.6)$ \\
\hline Triglycerides, mg/dL & $152.6(124.5)$ & II $9.7(90.7)$ & I $20.7(54.9)$ \\
\hline Metabolic syndrome**, \% & 15.6 & 5.9 & $\mathrm{n} / \mathrm{a}$ \\
\hline
\end{tabular}

Notes: *Outcomes are summarized as mean (SD) or percentage. **Metabolic syndrome was calculated as percentage of individuals with triglycerides $\geq \mathrm{I} 50 \mathrm{mg} / \mathrm{dL}$, $\mathrm{HDL}$ $\leq 40 \mathrm{mg} / \mathrm{dL}$, elevated blood pressure $>130 / 85 \mathrm{mmHg}$, and fasting glucose $\geq 100 \mathrm{mg} / \mathrm{dL}$ (central obesity unavailable). ${ }^{\wedge}$ Statistically significant difference as compared to cases; $\wedge \wedge$ institutional normal values were as follows: testosterone: $1.9-9.0 \mathrm{ng} / \mathrm{mL}$; free testosterone: $4.5-42.0 \mathrm{ng} / \mathrm{mL}$.

Abbreviations: HDL, high-density lipoprotein; LDL, low-density lipoprotein; n/a, not applicable.

\section{Cases and historical controls with similar numbers of tobacco users}

Cases and historical controls did not differ by age or smoking status (Table 1). Both cases and historical controls were overweight, with mean BMIs of $27.3 \pm 8.0 \mathrm{~kg} / \mathrm{m}^{2}$ and 27.8 $\pm 4.8 \mathrm{~kg} / \mathrm{m}^{2}$, respectively. Systolic blood pressure, diastolic blood pressure, and total cholesterol were comparable between the 2 groups. There was a trend toward higher TG levels among cases (mean: $152.6 \pm 124.5 \mathrm{mg} / \mathrm{dL}$ vs $120.7 \pm$ $54.9 \mathrm{mg} / \mathrm{dL}$ ); however, this trend was not statistically significant and was thought to be attributable to 1 outlier.

\section{Vascular testing of cases, recruited controls, and historical controls}

No statistically significant differences were found in LAE or SAE between cases and recruited controls or historical controls (Figure 1). Median LAE for cases was $17.0 \times 10$ (range: $14.0-37.5) \mathrm{mL} / \mathrm{mmHg}$ and $19.9 \times 10(10.9-23.3) \mathrm{mL} / \mathrm{mmHg}$ for recruited controls $(p=0.679)$. Median SAE for cases was $7.4 \times 100(6.8-14.0) \mathrm{mL} / \mathrm{mmHg}$ and $10.5 \times 100(7.0-14.0)$ $\mathrm{mL} / \mathrm{mmHg}$ for recruited controls $(p=0.086)$. Median LAE for historical controls was $18.6 \times 10 \mathrm{~mL} / \mathrm{mmHg}(13.3-25.8$ $\mathrm{mL} / \mathrm{mmHg} ; p=0.879$, compared to cases). Median SAE for historical controls was $9.8 \times 100 \mathrm{~mL} / \mathrm{mmHg}(6.1-12.0 \mathrm{~mL} /$ $\mathrm{mmHg} ; p=0.729$, compared to cases). When adjusted for age, BMI, and smoking status, the results continued to show no differences in SAE and LAE between cases and either the recruited controls or historical controls (all $p>0.6$ ).

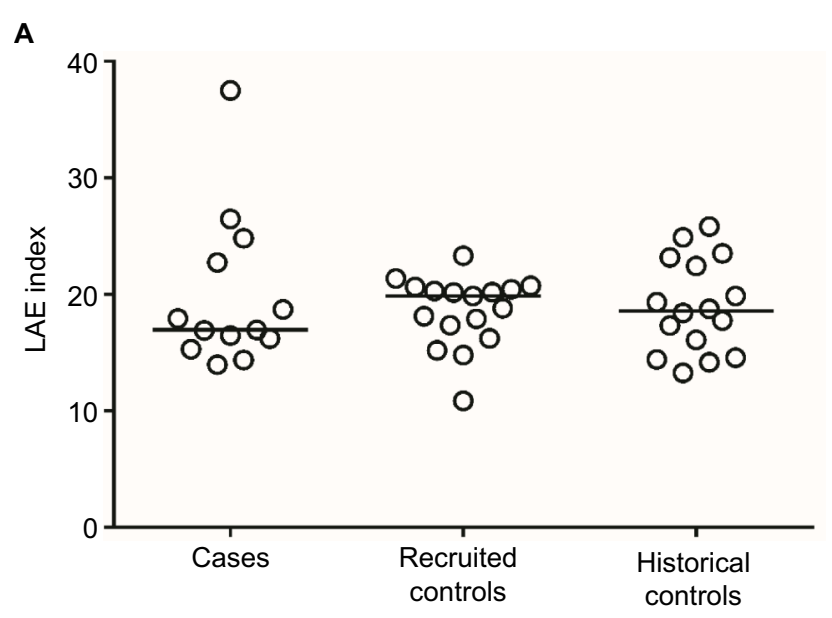

B

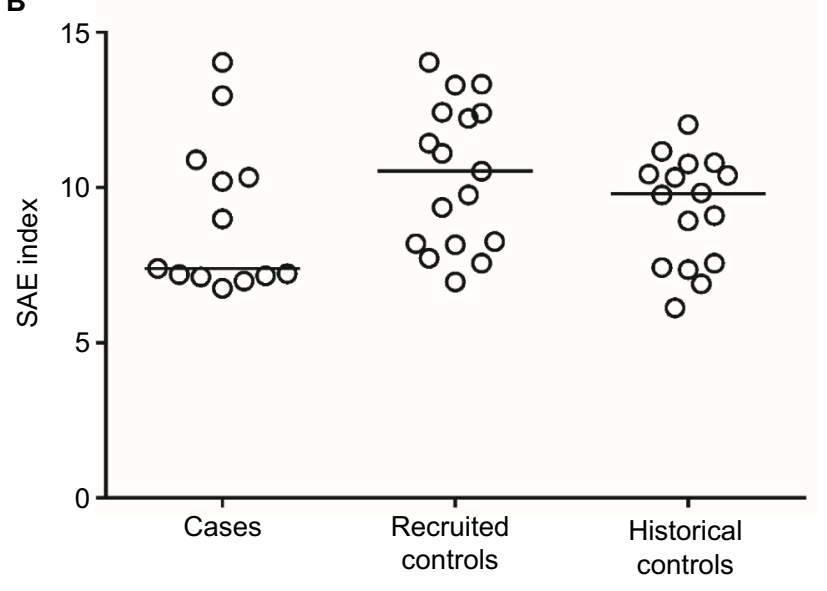

Figure I Distribution of LAE and SAE index values by disease status.

Note: Individual patient data (circle; average of 3 measures) and median (line) are presented. (A) LAE index; (B) SAE index.

Abbreviations: $L A E$, large artery elasticity; SAE, small artery elasticity. 
There was no difference in the median Endo-PAT reactive hyperemia peripheral arterial tonometry ratio between cases (ratio: 1.68) and controls (ratio: 1.54) $(p=0.574)$.

Given the small number of subjects, we were unable to evaluate the effect of time since treatment on vascular function testing.

\section{Vascular testing}

Risk factors for metabolic syndrome

A known risk factor for cardiovascular disease, metabolic syndrome is defined as $\geq 3$ of the following characteristics: central or abdominal obesity, TGs $\geq 150 \mathrm{mg} / \mathrm{dL}$, high-density lipoprotein $\leq 40 \mathrm{mg} / \mathrm{dL}$, elevated $\mathrm{BP}>130 / 85 \mathrm{mmHg}$, and fasting glucose $\geq 100 \mathrm{mg} / \mathrm{dL}$. Although metabolic syndrome was more common in cases (15\%) than in recruited controls $(6 \%)$, this difference was not statistically significant $(p=$ 0.565 ). Insufficient data on the characteristics of metabolic syndrome in our historical control group prevented direct comparisons with cases.

\section{Biomarkers of premature cardiovascular disease}

Biomarkers predictive of early vascular injury were assessed (Table 2). No statistically significant differences were seen between cases and recruited controls. Biomarker comparisons between cases and historical controls were not possible because data were not available for our historical controls.

\section{Discussion}

Although there may be a trend toward a reduction in SAE in testicular cancer survivors, there appears to be no clinically significant difference in SAE in survivors of testicular cancer treated with platinum-based chemotherapy as compared with controls 4 years after completing chemotherapy. Despite the risky behavior of tobacco use, we were unable to identify any difference in other cardiovascular risk factors (ie, metabolic syndrome and testosterone levels) between our cases and controls. In comparing our testicular cancer survivors to a group of tobacco-using controls, we also were unable to elucidate any difference in endothelial function.

Cisplatin can directly injure cardiac myocytes through oxidative stress and mitochondrial damage. ${ }^{10}$ In prior investigations, studies have reported quantitative evidence of endothelial dysfunction and damage in testicular cancer survivors. Nuver et $\mathrm{al}^{20}$ and van den Belt-Dusebout et $\mathrm{al}^{7}$ measured serum markers of acute inflammation, including vWF, tissue-type plasminogen activator, plasminogen activator inhibitor-1, fibrinogen, and high-sensitivity C-reactive protein. These biomarkers were measured before and 10 weeks after chemotherapy. Acute elevations in these markers were identified. Feldman et $\mathrm{al}^{21}$ also detected a reduction in SAE through endothelial reactivity at baseline and 34 weeks after chemotherapy $(p=0.07)$. Similarly, Dieckmann et a $1^{10}$ detected acutely increased vWF after cisplatin-based chemotherapy. The vWF values normalized with time, suggesting that direct endothelial damage may occur but that it stabilizes over time after chemotherapy. The data of Dieckmann et $\mathrm{a}^{10}$ support our hypothesis that the evidence of direct damage stabilizes over time. At 4 years after chemotherapy, as seen in our cohort, this damage seems to have normalized. Similarly, in a recent presentation using

Table 2 Primary measurements of vascular endothelium

\begin{tabular}{|c|c|c|c|c|c|}
\hline \multirow[t]{2}{*}{ Outcome } & \multicolumn{2}{|c|}{ Testicular cancer survivors $(N=\mid 3)$} & \multicolumn{2}{|c|}{ Recruited controls $(\mathrm{N}=17)$} & \multirow[t]{2}{*}{ p-value* } \\
\hline & $\mathbf{N}$ & Median (Min-Max) & $\mathbf{N}$ & Median (Min-Max) & \\
\hline \multicolumn{6}{|l|}{ Inflammation } \\
\hline hsCRP & 13 & I I 40 (4I-33882) & 17 & $731(8 I-13294))$ & 0.344 \\
\hline White blood cells & 13 & $5.5(3.1-9.1)$ & 17 & $5.2(3.5-7.0)$ & 0.379 \\
\hline \multicolumn{6}{|l|}{ Hemostasis } \\
\hline Fibrinogen & 13 & I57| (380-3736) & 17 & $\mid 876(608-282 \mid)$ & 0.178 \\
\hline Plasminogen activator inhibitor-I & 13 & $3108(2342-3772)$ & 17 & $2835(1679-4162)$ & 0.433 \\
\hline $\mathrm{t}-\mathrm{PA}$ & 13 & $19.5(12.1-137.1)$ & 17 & $28.2(11.4-60.3)$ & 0.143 \\
\hline \multicolumn{6}{|l|}{ Endothelial damage } \\
\hline vWF & 8 & $6.3(0.6)$ & 15 & $7.6(0.3-33.9)$ & 0.750 \\
\hline CECs & 13 & $1.0(0.0-8.0)$ & 17 & $1.0(0.0-1 \mathrm{I} .0)$ & 0.714 \\
\hline Surface VCAM-I & 13 & $67(0-100)$ & 16 & $67(0-100)$ & 0.616 \\
\hline Surface P-selectin & 13 & $40(13-83)$ & 17 & $60(20-100)$ & 0.202 \\
\hline Soluble VCAM-I & 13 & $1300(557-1592)$ & 17 & $995(542-2168)$ & 0.868 \\
\hline Soluble P-selectin & 13 & $42.0(29.7-54.4)$ & 17 & $4 I .6(34.4-63.7)$ & 0.709 \\
\hline
\end{tabular}

Note: *Wilcoxon rank-sum test (nonparametric); no adjustment.

Abbreviations: CEC, circulating endothelial cell; hsCRP, high-sensitivity C-reactive protein; Max, maximum; Min, minimum; t-PA, tissue-type plasminogen activator; VCAM, vascular cell adhesion molecule; vWF, von Willebrand factor. 
the Surveillance, Epidemiology, and End Results program, the risk of direct endothelial damage appears highest in the first year after chemotherapy. ${ }^{13,22}$ After chemotherapy, there is an increased cardiovascular mortality concentrated within Year 1 after testicular cancer diagnosis (standardized mortality ratio: $5.31, p<0.05)$. This risk decreases with time since diagnosis.

Cisplatin-based chemotherapy may also indirectly lead to cardiovascular disease. Studies suggest that cisplatin-based chemotherapy promotes an increase in risk factors for cardiovascular disease, namely, metabolic syndrome. ${ }^{2,23,24}$ Hyperlipidemia, central obesity, and insulin resistance have been previously described after cisplatin-based chemotherapy. ${ }^{14}$ In testicular cancer survivors, metabolic syndrome occurs in $20 \%-30 \%$ of survivors and occurs at an earlier age than expected, generally 3-5 years after therapy. ${ }^{24-26}$ Metabolic syndrome is a known risk factor for cardiovascular death. In our study, survivors had a trend toward a higher incidence of metabolic syndrome than our control populations. However, in our study, there was no statistical difference in metabolic syndrome between the cases and controls, and no difference in testosterone or free testosterone levels between cases and controls was detected; it is possible that the control of both testosterone levels and risk factors of metabolic syndrome explains why no changes in endothelial function were observed in our study.

Features of metabolic syndrome have care targets that can be intervened upon, such as hyperlipidemia reduction, weight management, and glucose control. These risk factors are modifiable in cancer survivors as well. Other studies suggest that low testosterone levels were associated with higher rates of metabolic syndrome (OR: 4.4). ${ }^{27}$ Additionally, tobacco use, well known to cause direct cardiovascular toxicity, ${ }^{28-30}$ was prevalent in our study in which $30 \%$ of our testicular cancer survivors smoked. Other studies have reported similar numbers in which tobacco use among young cancer survivors is highly prevalent, ${ }^{31-33}$ as well as being highly prevalent among testicular cancer survivors specifically. ${ }^{33}$ Addressing these modifiable risk factors is vital in caring for these cancer survivors. ${ }^{34}$

Our study is limited by its small sample size and low participation rate. If those survivors who did not respond to the study invitation were generally less healthy, this may have affected the study results, introducing some bias. This study is also limited by its cross-sectional nature in which longitudinal data are not available. Additionally, controlling for the discrepancy in race may be important, as other literature suggests that African Americans may have more arterial stiffness and changes in arterial elasticity at baseline ${ }^{35}$ the difference in race between our cohorts may account for the difficulty in interpreting these results. Given the suggestion of reduced SAE and the prevalence of risk factors for metabolic syndrome, longitudinal follow-up of this cohort is warranted. For future studies, however, we propose that interventions take into account race as well as address these modifiable risks factors at the time of completion of chemotherapy.

\section{Conclusion}

Previously published literature suggests that cisplatin-based chemotherapy causes direct endothelial toxicity. Our data do not support that endothelial damage exists at 4 years following completion of chemotherapy when there are no differences in testosterone levels or metabolic syndrome. Further follow-up, however, is needed. Long-term cardiovascular risk in testicular cancer survivors may be more related to indirect injury or secondary causes. Identifying these risk factors, such as metabolic syndrome, hypogonadism, and associated risky behaviors such as tobacco use, and intervening upon them needs to be done soon after the completion of chemotherapy. Collaboration with other health-care providers will likely be necessary in order to modify these long-term risk factors.

\section{Acknowledgments}

The results of this study were presented at the meeting of the American Society of Clinical Oncology in June 2014 as a poster presentation and published in abstract form in the Journal of Clinical Oncology. We greatly appreciate Dr Douglas Yee, for his time and effort in discussing the concept and providing insight on the overall project, as well as Dr Rachel Lerner and Dr Dylan Zylla, for their time and assistance in helping recruit subjects at the Park Nicollet Health Services. Finally, we thank Dr Jason Baker for sharing data on historical controls, as well as Natalia Florea for her contributions in performing vascular assessments. We also thank the following sources of funding for our project: AFLAC Young Investigator Award in Young Adult Oncology and Building Interdisciplinary Research Careers in Women's Health (National Institutes of Health grant number K12-HD055887).

\section{Disclosure}

The authors report no conflicts of interest in this work. 


\section{References}

1. Shanmugalingam T, Soultati A, Chowdhury S, Rudman S, Van Hemelrijck M. Global incidence and outcome of testicular cancer. Clin Epidemiol. 2013;5:417-427.

2. Haugnes HS, Wethal T, Aass N, et al. Cardiovascular risk factors and morbidity in long-term survivors of testicular cancer: a 20-year followup study. J Clin Oncol. 2010;28(30):4649-4657.

3. Vaughn DJ, Palmer SC, Carver JR, Jacobs LA, Mohler ER. Cardiovascular risk in long-term survivors of testicular cancer. Cancer. 2008;112(9):1949-1953.

4. Hashibe M, Abdelaziz S, Al-Temimi M, et al. Long-term health effects among testicular cancer survivors. J Cancer Surviv. 2016;10(6): 1051-1057.

5. Alanee SR, Feldman DR, Russo P, Konety B. Long-term mortality in patients with germ cell tumors: effect of primary cancer site on cause of death. Urol Oncol. 2014;32(1):26.e9-e15.

6. van den Belt-Dusebout AW, de Wit R, Gietema JA, et al. Treatment-specific risks of second malignancies and cardiovascular disease in 5-year survivors of testicular cancer. J Clin Oncol. 2007;25(28):4370-4378.

7. van den Belt-Dusebout AW, Nuver J, de Wit R, et al. Long-term risk of cardiovascular disease in 5-year survivors of testicular cancer. J Clin Oncol. 2006;24(3):467-475.

8. Huddart RA, Norman A, Shahidi M, et al. Cardiovascular disease as a long-term complication of treatment for testicular cancer. J Clin Oncol. 2003;21(8):1513-1523.

9. Travis LB, Beard C, Allan JM, et al. Testicular cancer survivorship: research strategies and recommendations. J Natl Cancer Inst. 2010;102(15):1114-1130.

10. Dieckmann KP, Gerl A, Witt J, Hartmann JT; German Testicular Cancer Study Group. Myocardial infarction and other major vascular events during chemotherapy for testicular cancer. Ann Oncol. 2010;21(8):1607-1611.

11. Willemse PM, Burggraaf J, Hamdy NA, Osanto S. Reply: 'Comment on Prevalence of the metabolic syndrome and cardiovascular disease risk in chemotherapy-treated testicular germ cell tumour survivors'. $\mathrm{Br}$ J Cancer. 2013;109(9):2503-2504.

12. Nuver J, Smit AJ, van der Meer J, et al. Acute chemotherapy-induced cardiovascular changes in patients with testicular cancer. J Clin Oncol. 2005;23(36):9130-9137.

13. Fung C, Fossa SD, Milano MT, Sahasrabudhe DM, Peterson DR, Travis LB. Cardiovascular disease mortality after chemotherapy or surgery for testicular nonseminoma: a population-based study. J Clin Oncol. 2015;33(28):3105-3115.

14. Lubberts $\mathrm{S}$, Boer $\mathrm{H}$, Altena $\mathrm{R}$, et al. Vascular fingerprint and vascular damage markers associated with vascular events in testicular cancer patients during and after chemotherapy. Eur J Cancer. 2016;63: $180-188$

15. Baker JV, Duprez D, Rapkin J, et al. Untreated HIV infection and large and small artery elasticity. J Acquir Immune Defic Syndr. 2009;52(1): 25-31.

16. Blaes A, Beckwith H, Florea N, et al. Vascular function in breast cancer survivors on aromatase inhibitors: a pilot study. Breast Cancer Res Treat. 2017;166(2):541-547.

17. Grey E, Bratteli C, Glasser SP, et al. Reduced small artery but not large artery elasticity is an independent risk marker for cardiovascular events. Am J Hypertens. 2003;16(4):265-269.

Vascular Health and Risk Management

\section{Publish your work in this journal}

Vascular Health and Risk Management is an international, peerreviewed journal of therapeutics and risk management, focusing on concise rapid reporting of clinical studies on the processes involved in the maintenance of vascular health; the monitoring, prevention and treatment of vascular disease and its sequelae; and the involvement of
18. Duprez DA, Hearst MO, Lutsey PL, et al. Associations among lung function, arterial elasticity, and circulating endothelial and inflammation markers: the Multiethnic Study of Atherosclerosis. Hypertension. 2013;61(12):542-548

19. Rubinshtein R, Kuvin JT, Soffler M, et al. Assessment of endothelial function by non-invasive peripheral arterial tonometry predicts late cardiovascular adverse events. Eur Heart J. 2010;31(9):1142-1148.

20. Nuver J, Smit AJ, Sleijfer DT, et al. Microalbuminuria, decreased fibrinolysis, and inflammation as early signs of atherosclerosis in long-term survivors of disseminated testicular cancer. Eur J Cancer. 2004;40(5):701-706.

21. Feldman DR, Jacobsen EP, Woo K, et al. Acute changes in endothelial function with cisplatin among germ cell tumor patients. J Clin Oncol. 2014;32:5s (Suppl; Abstr 9587).

22. Fung C, Sesso HD, Williams AM, et al; Platinum Study Group. Multiinstitutional assessment of adverse health outcomes among North American testicular cancer survivors after modern cisplatin-based chemotherapy. J Clin Oncol. 2017;35(11):1211-1222.

23. de Haas EC, Zwart N, Meijer C, et al. Association of PAI-1 gene polymorphism with survival and chemotherapy-related vascular toxicity in testicular cancer. Cancer. 2010;116(24):5628-5636.

24. de Haas EC, Altena R, Boezen HM, et al. Early development of the metabolic syndrome after chemotherapy for testicular cancer. Ann Oncol. 2013;24(3):749-755.

25. Willemse PP, van der Meer RW, Burggraaf J, et al. Abdominal visceral and subcutaneous fat increase, insulin resistance and hyperlipidemia in testicular cancer patients treated with cisplatin-based chemotherapy. Acta Oncol. 2014;53(3):351-360.

26. Richie JP. Reply: 'Early development of the metabolic syndrome after chemotherapy for testicular cancer'. J Urol. 2014;191(4):987.

27. Bogefors C, Isaksson S, Bobjer J, et al. Hypogonadism in testicular cancer patients is associated with risk factors of cardiovascular disease and the metabolic syndrome. Andrology. 2017;5(4):711-717.

28. Agha G, Loucks EB, Tinker LF, et al. Healthy lifestyle and decreasing risk of heart failure in women: the Women's Health Initiative Observational Study. J Am Coll Cardiol. 2014;64(17):1777-1785.

29. Schnohr P, Marott JL, Kristensen TS, et al. Ranking of psychosocial and traditional risk factors by importance for coronary heart disease: the Copenhagen City Heart Study. Eur Heart J. 2015;36(22):1385-1393.

30. Cheng S, Claggett B, Correia AW, et al. Temporal trends in the population attributable risk for cardiovascular disease: the Atherosclerosis Risk in Communities Study. Circulation. 2014;130(10):820-828.

31. Tarleton HP, Ryan-Ibarra S, Induni M. Chronic disease burden among cancer survivors in the California Behavioral Risk Factor Surveillance System, 2009-2010. J Cancer Surviv. 2014;8(3):448-459.

32. Karam-Hage M, Cinciripini PM, Gritz ER. Tobacco use and cessation for cancer survivors: an overview for clinicians. CA Cancer J Clin. 2014;64(4):272-290.

33. Reilley MJ, Jacobs LA, Vaughn DJ, Palmer SC. Health behaviors among testicular cancer survivors. J Community Support Oncol. 2014;12(4):121-128.

34. Smith WA, Li C, Nottage KA, et al. Lifestyle and metabolic syndrome in adult survivors of childhood cancer: a report from the St. Jude Lifetime Cohort Study. Cancer. 2014;120(17):2742-2750.

35. Wassel CL, Jacobs DR Jr, Duprez DA, et al. Association of selfreported race/ethnicity and genetic ancestry with arterial elasticity: the Multi-Ethnic Study of Atherosclerosis (MESA). J Am Soc Hypertens. 2011;5(6):463-472.

Dovepress

metabolic disorders, particularly diabetes. This journal is indexed on PubMed Central and MedLine. The manuscript management system is completely online and includes a very quick and fair peer-review system, which is all easy to use. Visit http://www.dovepress.com/ testimonials.php to read real quotes from published authors. 